\title{
Magnetic field evolves to gravity field part:5 Final
}

\author{
John C. Hodge ${ }^{1 *}$ \\ ${ }^{1}$ Retired, 477 Mincey Rd., Franklin, NC, 28734
}

November 25, 2019

\begin{abstract}
The Scalar Theory of Everything (STOE) suggests gravity emerges from magnetic effects of hods in matter structures. An experiment is performed to demonstrate the net magnetic field from hods in a body only attracts the hods from other masses to evolve into the gravity affect for distances greater than molecular sizes.
\end{abstract}

keywords: magnet, gravity, atomic structure, atomic spectra

\section{INTRODUCTION}

The Scalar Theory of Everything (STOE) started with the Quasi Steady State Cosmology (QSSC) model that suggested the creation of matter at the center of galaxies (Sources) (Narlikar 2002; Narlikar et al. 2015). This model is successful at explaining spiral galaxy rotation curves without dark matter and other problems for traditional cosmology. The STOE adds Sinks at the center of elliptical galaxies. The STOE uses the Newtonian concept of forces rather than energy to develop a Universal Equation (Hodge 2018e). These additions allow the description of many more anomalous and problem observations.

The STOE posits the universe is composed of hods and plenum which emerge to form all in the universe (Hodge 2016b). The hods are discrete and the plenum is continuous (Hodge 2018e, figure 3.1). That is, discrete phenomena emerge from hod behavior. Continuous phenomena emerge from plenum behavior.

The STOE proposes that phenomena of the small may be better explained by cause-and-effect analogy to our everyday world. Because the universe is one, the models valid in our everyday world apply to the small (quantum) world. The STOE model of mass is an assembly of hods.

The STOE developed a model of photons and photon behavior. This model and its simulation program of photons successfully reproduced the interference experiments including those that rejected wave models of light (Hodge project).

*E-mail: jchodge@frontier.com 
A model of gravity requires a link between cosmology and the small world. The STOE model of gravity is similar to the model of magnetic effects. That is, the forces are a result of the divergence of the plenum density on hods.

The STOE experiments rejected the Biot-Savart Law and suggested two types of magnetic effects (Hodge 2018b,c,d). One type emanates from permanent magnets and hods, the other from electric currents. The model of hods includes the idea the hods causes a plenum density that mimics the North and South poles of permanent magnets. The forces of the magnetic poles are different for the different poles (Hodge 2019a).

Hodge (2016a) suggest particles are specific structures of hods (Hodge 2016a, 2019a). Hodge (2019b) suggest the atomic structure is an assembly of photons which hold the electrons in specific structures. This in contrast to the wave or quantum models to account for spectrographic observations.

This Paper suggests a STOE compatible model of gravity based on the interaction of the magnetic fields of hods in one body of mass with hods in another body of mass. The description of the basic model is in section 2. An interpretation of the experiment is in section 3. The Discussion and Conclusion are in section 4.

\section{THE EXPERIMENT}

The same fixture and equipment used in Hodge (2019a) was used in this experiment. The bottom support had attached several disk magnets. The upper support had the same number of disk magnets as the bottom support and held so that they can rotate about a center. The center was held on the axis of the bottom magnets. The North Pole was the zero of the angle measured $A$ and was up at $A=0^{\circ}$. The force $F$ exerted on the lower magnet was measured by the scale for various position of rotation of the upper magnet. The magnets have a diameter $L=18 \mathrm{~mm}$.

An asymmetry was indicated for Zone 2 of Hodge (2019a) which was the distance $d$ between magnets for $d>2 L$. The Zone 2 force was proportional to the square of the inverse distance. When 1 or few magnets were used, the $F$ readings were too low. With seven magnets the $d=97 \mathrm{~mm}(d>5 L)$ gave acceptable $F$ readings for the scale used.

Figure 1 shows the results of an average of 10 runs of the present experiment. Each run consisted of a disassembly and reassembly of the measuring fixture. The same magnets were used for all 10 runs.

Region 2 is the area of the graph where the North Poles of the upper and lower magnets face each other (therefore, the $F$ is repulsive, positive reading). Region 1 is the area not in Region 2 (attractive poles - negative $F$ - and repulsive South poles). The equation for the lines is:

$$
F=C \cos (A),
$$

where for Region 1 the coefficient $C_{\mathrm{S} 1}=1.5 \pm 0.1 \mathrm{gw}, C_{\mathrm{N} 1}=-1.5 \pm 0.1 \mathrm{gw}$ which are shown as a solid line on the graph. For Region $2 C_{\mathrm{N} 2}=-1.3 \pm 0.1 \mathrm{gw}$ which 


\section{THE EXPERIMENT}

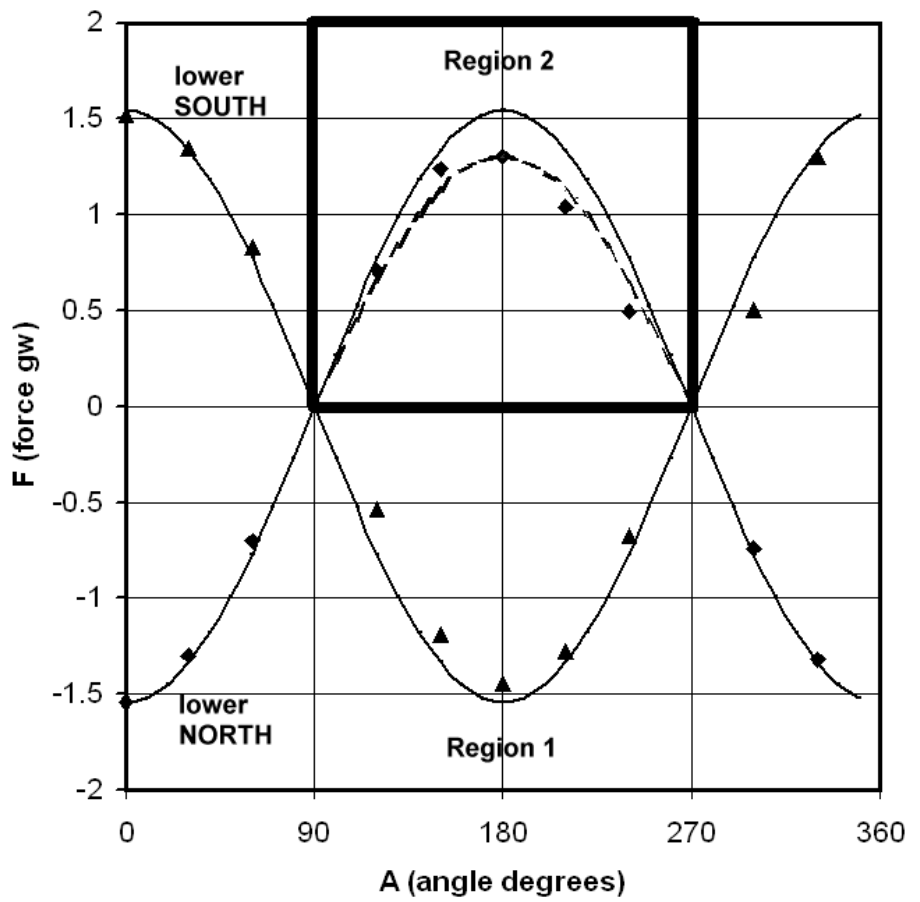

Figure 1: Graph of the results. The diamonds indicate the data points for the case of the lower magnet with the North Pole up. The triangles indicate the data points for the case of the lower magnet with the South Pole up. 

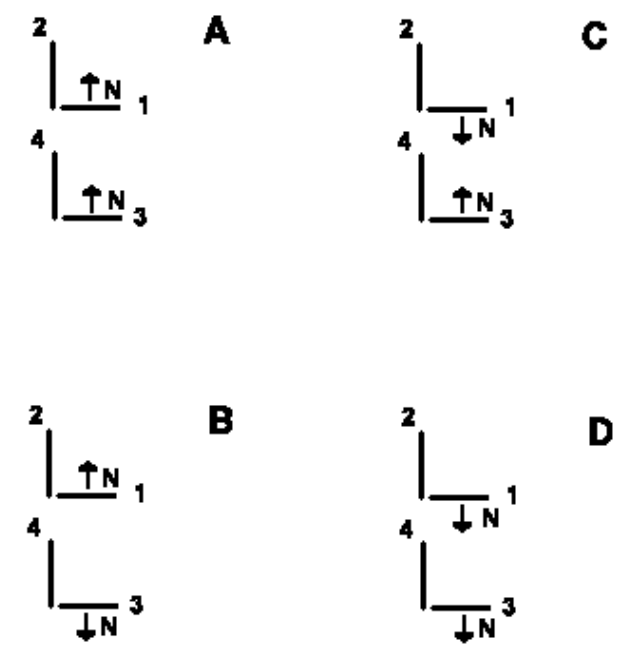

B

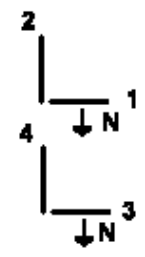

D

Figure 2: Sketches of the various hod configurations of hods in two bodies.

is shown as a dashed line on the graph. The uncertainty is determined by the standard deviation of the 10 repetitions of the experiment.

\section{INTERPRETATION}

The STOE suggests all matter is composed of hods that have a permanent magnetic field that causes their influence on the plenum. The disk magnets in the experiment are the Newtonian world analogy of the hods. The measurement is the magnetic force exerted by one hod on another. If the magnets are close, a mass of a body is formed. If the distance is such that there are two bodies, the very weak magnetic force is only attractive and is called "gravity".

A 2-dimension situation is simplified for ease of description in Fig. 2 This figure depicts the four cases where the lines represent hods and the numbers identify the hods. Hods " 1 " and "2" are in one mass, hods " 3 " and " 4 " is another mass. Hods " 2 " and " 4 " are at an $A=90^{\circ}$ or $A=270^{\circ}$ which exerts $F=0$ on each mass. Hods " 1 " and " 3 " are at an $A=0^{\circ}$ or $A=180^{\circ}$ which exerts $F=C$ on each mass. Cases "A" and "D" are attractive in Region 1. Case " $\mathrm{B}$ " is repulsive in Region 1. Case " $\mathrm{C}$ " is repulsive in Region2.

The total Force $F_{\mathrm{T}}$ exerted on each mass is the sum of these four situations: $F=-1.5+1.5+1.3-1.5=-0.2 \mathrm{gw}$, respectively. 


\section{DISCUSSION AND CONCLUSION}

Experiments with disk magnets demonstrate closeness may determine structure. Adjacent disks in near contact must be either axially or perpendicular such as seen in photons and electrons. However, farther apart magnets can exist without joining. This will place limits on the structure of particles, nuclei, atoms, and molecules. These structures provide a random orientation of hods in a body consisting of many molecules.

Note in Fig. 2 there are only two hods in each mass, but only one has the force $F_{\mathrm{h}}$ of the hod directed to the other mass because $A=0^{\circ}$. Remember $F_{\mathrm{h}}$ is different for the poles. The other hod has $A=90^{\circ}$ or $A=270^{\circ}$. The general case for a mass would have the $F=\sqrt{2} F_{\mathrm{h}} / N d_{\text {avg }}^{2}$ where $N$ is the number of hods in the 2-dimensional body and $d_{\text {avg }}$ is the average distance of the hods (the center of mass). A 3-dimensional body has $F=\sqrt{3} F_{\mathrm{h}} / N d_{\text {avg }}^{2}$ in general.

However there are exceptions. One is the column of hods that is a photon. The photon has the $F_{\mathrm{h}} N \cos (A)$ oriented in the axial direction. The direction perpendicular and in the plane with the center of the photon has $F=0$. The neutrino structure two axis with the $F=\sqrt{2} F_{\mathrm{h}} / N$ and one axis with $F=0$. A body that has permanent magnetic properties has the assumption of random orientation being invalid.

An experiment is performed to demonstrate the magnetic field from hods attracts the hods from other masses to evolve into the gravity affect.

\section{ORCID iD}

John C. Hodge: https://orcid.org/0000-0002-1520-2153

\section{References}

Hodge, J.C., 2012, Photon diffraction and interference, IntellectualArchive, Vol.1, No. 3, P. 20,. http://intellectualarchive.com/?link=item\&id=597

Hodge, J.C., 2016a, Structure and spin of the neutrino, electron, and positron, IntellectualArchive, Vol.5, No. 5, P. 1,. http://intellectualarchive.com/?link=item\&id=1694

Hodge, J.C., 2016b, STOE emergence, http://intellectualarchive.com/?link=item\&id=1757

Hodge, J.C., 2017, STOE simulation of photon spectrographic behavior, IntellectualArchive, Vol.6, No. 6, P. 1, http://intellectualarchive.com/?link=item\&id $=1884$

Hodge, J.C., 2018a, STOE electric charge, IntellectualArchive, Vol.7, No. 2, P 1. 1 , http://intellectualarchive.com/?link=item\&id=1917 


\section{REFERENCES}

Hodge, J.C., 2018b, Magnetostatics relation to gravity with experiment that rejects Biot-Savart Law, IntellectualArchive, Vol.7, No. 3, P. 1,. http://intellectualarchive.com/?link=item\&id=1945

Hodge, J.C., 2018c, Another experiment rejects Ampere's Law and supports the STOE model, IntellectualArchive, Vol.7, No. 4, P. 6,. http://intellectualarchive.com/?link=item\&id=1956

Hodge, J.C., 2018d, Two different types of magnetic field, IntellectualArchive, Vol.4, No. 4, P. 1,. http://intellectualarchive.com/?link=item\&id=1964

Hodge, J.C., 2018e, STOE replaces relativity and quantum mechanics, (ISBN 978-613-9-91465-4, (LAP LAMBERT Acedemic publishing, Mauritius, available through Amazon.com).

Hodge, J.C., 2019a, Magnetic field evolves to gravity field part:1 Repulsion, IntellectualArchive, Vol.8, No. 3, P. 17,. http://intellectualarchive.com/?link=item\&id=2164

Hodge, J.C., 2019b, Magnetic field evolves to gravity field part:2 Particles, IntellectualArchive, Vol.8, No. 3, P. 30,. http://intellectualarchive.com/?link $=$ item\&id $=2170$

Hodge, J.C., 2019d, Magnetic field evolves to gravity field part:4 Atomic structure , IntellectualArchive, Vol.8, No. 4, P. 1 ,. http://intellectualarchive.com/?link=item\&id $=2199$

Hodge, J.C., Project, STOE photon diffraction and interference , https://www.researchgate.net/project/STOE-photon-diffraction-andinterference .

Narlikar, J.V., 2002, An introduction to Cosmology, (ISBN 052179376 9), (Cambridge University Press)

Narlikar, J.V., et al. 2015, Gravitational wave background in the Quasi-Steady Cosmology, Mon. Not. Astron. Soc., 451, 2, P. 1390 